\title{
Depletion of hsa_circ_0000 I44 Suppresses Oxaliplatin Resistance of Gastric Cancer Cells by Regulating miR-502-5p/ADAM9 Axis
}

\author{
Haifeng Gao',* \\ Jiajia $\mathrm{Xu}^{2}$,* \\ Fen $\mathrm{Qiao}^{3}$ \\ Liangjun $\mathrm{Xue}^{4}$
}

'Department of Clinical Laboratory, Baoji Central Hospital, Baoji City, 721008, Shaanxi Province, People's Republic of China; ${ }^{2}$ Department of Organic Chemistry and Pharmaceutical

Chemistry, Pharmaceutical College of Guangxi Medical University, Nanning,

Guangxi, People's Republic of China; ${ }^{3}$ Department of Pediatrics, Baoji Central Hospital, Baoji City, Shaanxi Province, People's Republic of China; ${ }^{4}$ Department of Radiotherapy, Yijishan Hospital of Wannan Medical College, Wuhu City, Anhui Province, 24I00I, People's

Republic of China

*These authors contributed equally to this work
Correspondence: Liangjun Xue Department of Radiotherapy, Yijishan Hospital of Wannan Medical College, No.

2 Zheshan West Road, Wuhu City, Anhui Province, 24I00I, People's Republic of China

Tel +86553-5738279

Email xuelj0I6@I63.com
Background: Circular RNAs (circRNAs) have been disclosed to exert important roles in human cancers, including gastric cancer (GC). CircRNA hsa_circ_0000144 was identified as an oncogene in GC development. The aim of our study was to explore the role of hsa_circ_0000144 in oxaliplatin (OXA) resistance of GC.

Methods: Expression levels of hsa_circ_0000144, microRNA-502-5p (miR-502-5p) and A disintegrin and metalloproteinase 9 (ADAM9) were examined by quantitative real-time PCR (RT-qPCR) or Western blot assay. The OXA resistance of GC cells was evaluated by Cell Counting Kit-8 (CCK-8) assay. Colony formation assay was performed to assess the colony formation capacity. Cell apoptosis was determined by flow cytometry and caspase 3 activity. And cell migration and invasion were detected by Transwell assay. Target association between miR-502-5p and hsa_circ_0000144 or ADAM9 was demonstrated by dualluciferase reporter assay and RNA immunoprecipitation (RIP) assay. Moreover, role of hsa_circ_0000144 in vivo was analyzed by xenograft tumor assay.

Results: Hsa_circ_0000144 and ADAM9 were highly expressed, while miR-502-5p was downregulated in OXA-resistant GC tissues and cells. Depletion of hsa_circ_0000144 could inhibit OXA resistance, proliferation and metastasis in OXA-resistant GC cells, which was attenuated by miR-502-5p inhibition. Hsa_circ_0000144 sponged miR-502-5p to positively regulate ADAM9 expression. MiR-502-5p suppressed OXA resistance, proliferation and metastasis in OXA-resistant GC cells by targeting ADAM9. Hsa_circ_0000144 knockdown could hamper tumor growth in vivo.

Conclusion: Hsa_circ_0000144 exerted inhibitory effects on OXA resistance, proliferation and metastasis of OXA-resistant GC cells by regulating miR-502-5p/ADAM9 axis, at least in part. Keywords: GC, OXA resistance, hsa_circ_0000144, miR-502-5p, ADAM9, OXA-resistant GC cells

\section{Introduction}

Gastric cancer (GC) is a serious threat to human health and wealth, ranking as the most common subtype and the most lactiferous cancer among malignancies derived from gastrointestinal system. ${ }^{1}$ Unhealthy dietary habits and infection with Helicobacter pylori are the main risk factors of developing $\mathrm{GC}^{2}$ At present, gastrectomy and chemotherapy remain to be major approaches for GC treatment. But occurrence of drug resistance significantly reduced therapeutic efficiency. ${ }^{3}$ Oxaliplatin (OXA) is a broadly used anticancer medicine worldwide. ${ }^{4}$ Therefore, clarifying the molecular mechanisms associated with GC progression and chemoresistance development is urgently needed. 
Circular RNAs (circRNAs) are a novel category of non-coding RNAs (ncRNAs), characterized by the special covalently closed loop structure. ${ }^{5}$ CircRNAs have been discovered to function in proliferation, apoptosis and metastasis of GC cells, as well as to act as diagnostic biomarkers. ${ }^{6}$ NcRNAs, including long noncoding RNAs (lncRNAs) and circRNAs, were reported to have close association with GC chemoresistance. ${ }^{7}$ For example, circRNA_001569 facilitated GC cell growth by sponging miR-145. ${ }^{8}$ CircRNA circ-PVT1 promoted paclitaxel resistance of GC cells by sponging miR-124$3 p$ to increase ZEB1 expression. ${ }^{9}$ Moreover, circ_0001546 could suppress GC cell proliferation and OXA resistance by absorbing miR-421 and activating ATM/Chk2/p53-dependent pathway. ${ }^{10}$ Derived from SLAM family member 6 (SLAMF6), hsa_circ_0000144 (Location: chr1:160472466-160472794; spliced sequence length: $328 \mathrm{bp}$ ) was demonstrated to be an oncogene in GC progression. ${ }^{11}$ However, the role of hsa_circ_0000144 in GC OXA resistance remains to be investigated.

MicroRNAs (miRNAs) are also ncRNAs, with diverse regulatory roles in multicellular organisms. ${ }^{12}$ MiRNAs were identified as biomarkers for GC chemoresistance, with potential applicability for GC diagnosis and therapy. ${ }^{13}$ MiR-502-5p participated in GC development through modulating IRF-1 expression. ${ }^{14}$ Circular RNA Interactome predicted miR-502-5p to be a target of hsa_circ_0000144. Here, we intended to explore the functional effects of miR-502-5p on OXA resistance of GC cells.

A disintegrin and metalloproteinase 9 (ADAM9) is a member of the ADAM family, which has the potential to be a therapeutic target for human cancers. ${ }^{15}$ ADAM9 is involved in intercellular interactions and degradation of extracellular matrix (ECM). ${ }^{16}$ ADAM9 was evidenced to be upregulated in GC tissues and cells, and played protumor role in $\mathrm{GC} .{ }^{17}$ Moreover, ADAM9 was predicted to be a target mRNA of miR-502-5p by TargetScan. The involvement of hsa_circ_0000144/miR-502-5p/ADAM9 pathway in GC OXA resistance and development needs to be corroborated.

In this work, OXA-resistant GC cell lines were structured. The dysregulation of hsa_circ_0000144 in OXAresistant GC tissues and cells was determined. Functional assays in vitro and in vivo were performed to explore the role of hsa_circ_0000144 in GC progression, as well as the potential mechanism.

\section{Materials and Methods}

\section{Patients and Tissues}

Prior to this study, we acquired permission from the Ethic Committee of Baoji Central Hospital. Fifty pairs of GC tissues and matched normal tissues were collected from patients diagnosed with GC at Baoji Central Hospital. All tumor tissues were classified as OXA-sensitive (25 cases) and OXA-resistant (25 cases) on the basis of Revised RECIST guideline (version 1.1). ${ }^{18}$ Clinical samples were kept at $-80^{\circ} \mathrm{C}$ until used. All patients submitted written informed consents.

\section{Cell Culture and Transfection}

Human gastric mucosal cell line GES-1 (CL-0563; Procell, Wuhan, China), GC cell lines AGS (CRL-1739; Procell) and MKN45 (CL-0292; Procell) were cultured in Roswell Park Memorial Institute (RPMI)-1640 medium (Invitrogen, Carlsbad, CA, USA) supplemented with $10 \%$ fetal bovine serum (v/v) (Gibco, Gran Island, NY, USA) and $1 \%$ penicillin/streptomycin (Invitrogen) in a $37^{\circ}$ $\mathrm{C}$ humidified incubator containing $5 \% \mathrm{CO}_{2}$. To construct OXA-resistant cell lines (AGS/OXA and MKN45/OXA), MKN45 and AGS cells were exposed treated with gradually increasing concentrations of OXA (Solarbio, Beijing, China) for 6 months as previously described. ${ }^{19}$ To maintain OXA resistance, medium was added with OXA (12.4 $\mu \mathrm{M}$ for AGS/OXA and 18.6 $\mu \mathrm{M}$ for MKN45/OXA).

Small interfering RNAs (siRNAs) against hsa circ_0000144 (si-hsa_circ_0000144\#1, 5'-AGGGAGAG AGAGGTAGAACTA-3', si-hsa_circ_0000144\#2, 5'-AA GGGAGAGAGAGGTAGAACT-3' and si-hsa _circ_0000144\#3, 5'-GAAAGGGAGAGAGA GGTAGAA-3'), miR-502-5p mimic (miR-502-5p, 5'AUCCUUGCUAUCUGGGUGCUA-3'), miR-502-5p inhibitor (anti-miR-502-5p, 5'-UAGGAACGAUAGACC CACGAU-3') and overexpression vector of ADAM9 (pcDNA-ADAM9), as well as their corresponding negative controls (si-NC, 5'-GGACUCUCGGAUUGUAAGAUU-3', NC, 5'-ACGUGACACGUUCGGAGAA-3', anti-NC, 5'UGAGCUGCAUAGAGUAGUGAUUA- ${ }^{\prime}$ and pcDNANC) were synthesized by GenePharma (Shanghai, China). In addition, overexpression plasmid of hsa_circ_0000144 harboring its full length (hsa_circ_0000144) and negative control (circ-NC) were established using pCD5-ciR vector by Geneseed Biotech Co., Ltd. (Guangzhou, China). AGS/OXA and MKN45/OXA cells were transfected with oligonucleotides or plasmids using Lipofectamine 3000 (Invitrogen) as 
instructed by the manufacturer. After $48 \mathrm{~h}$, transfected OXAresistant GC cells were subjected for further investigation.

\section{Quantitative Real-Time PCR (RT-qPCR)}

Total RNA derived from clinical samples or cultured cells was isolated using TRIzol Reagent (Invitrogen), then reverse transcribed into cDNA exploiting GoScript Reverse Transcription System (Promega, Madison, WI, USA) or miScript Reverse Transcription Kit (Qiagen, Germantown, MD, USA). As the user's manual directed, SYBR Green Realtime PCR Master Mix (Solarbio) was applied to evaluate the relative expression of hsa circ_0000144 and mRNA, normalized to $18 \mathrm{~S}$ rRNA. Additionally, all-in-one miRNA RT-qPCR Detection Kit (GeneCopoeia Inc., Rockville, MD, USA) was utilized to detect miRNAs expression, normalized to U6 snRNA. Relative expression of genes was calculated by $2^{-\Delta \Delta C t}$ formula. ${ }^{20}$ All qPCR primers were exhibited in Table 1.

\section{Nuclear/Cytoplasmic Fractionation}

The current assay was used to validate the intracellular localization of hsa_circ_0000144. Nuclear and cytoplasmic fractions were isolated using PARIS ${ }^{\mathrm{TM}}$ Kit (Thermo Fisher Scientific, Waltham, MA, USA) in conformity to the recommended instructions. ${ }^{21}$ Moreover, U6 and $18 \mathrm{~S}$ rRNA served as internal references for nuclear and cytoplasmic fractions, separately.

\section{RNase R Treatment}

To examine the stability of hsa_circ_0000144 in OXAresistant GC cells, $10 \mu \mathrm{g}$ total RNA was incubated with RNase R (40 U; Epicentre Biotechnologies, Madison, WI, USA) or not. At $1 \mathrm{~h}$ post digestion, the enrichments of hsa_circ_0000144 and linear mRNA (SLAMF6) were analyzed using RT-qPCR assay.

\section{Drug Resistance Assay}

AGS, MKN45, AGS/OXA and MKN45/OXA cells in a medium supplemented with $10 \%$ serum $\left(5 \times 10^{3}\right.$ per well) were separately plated into 96 -well plates. Twentyfour hours later, medium containing OXA at different concentrations $(5,10,20,40,80,160$ or $320 \mu \mathrm{M})$ was added to replace the complete medium. At $48 \mathrm{~h}$ post incubation, cell counting kit $8(\mathrm{CCK}-8)$ reagent $(10 \mu \mathrm{L}$; Beyotime, Shanghai, China) was instilled into each well. After another $2 \mathrm{~h}$, the absorbance at $450 \mathrm{~nm}$ was recorded using a microplate reader (Bio-Rad, Hercules, CA, USA).
Subsequently, the survival curves were drawn, and the half inhibition concentration (IC50) was estimated.

\section{Colony Formation Assay}

For colony formation assay, transfected AGS/OXA and MKN45/OXA cells $\left(5 \times 10^{2}\right)$ were seeded into 6-well plates. After 15-d routine culture, generated colonies were fastened using 4\% paraformaldehyde (Beyotime) and dyed using crystal violet (Beyotime), then counted utilizing Image J software (NIH, Bethesda, MD, USA) and photographed.

\section{Cell Apoptosis Analysis}

To monitor cell apoptosis, the Annexin V-fluorescein isothiocyanate (FITC) Apoptosis Detection kit (BD Biosciences, Franklin Lakes, NJ, USA) was applied. In brief, transfected AGS/OXA and MKN45/OXA cells were harvested and then incubated with Annexin V-FITC and propidium iodide (PI) in the dark for staining. Then, apoptotic cells were identified utilizing a flow cytometer (BD Biosciences).

Caspase 3 is a key mediator of cell apoptosis, whose activation is essential for apoptotic events and cell death. ${ }^{22}$ Here, caspase-3 Activity Assay Kit (ab252897; Abcam, Cambridge, MA, USA) was used to evaluate the caspase 3 activity in AGS/OXA and MKN45/OXA cells referring to the specifications.

\section{Transwell Migration and Invasion Assays}

For migration analysis, $1 \times 10^{4}$ transfected AGS/OXA and MKN45/OXA cells in medium without serum were plated in the upper chambers ( $8 \mu \mathrm{m}$; BD Biosciences). Meanwhile, complete medium was added into the lower ones. Twenty-four hours later, non-migrated cells were discarded. Cells migrated through fibronectin-coated polycarbonate membrane were subjected to immobilization using paraformaldehyde (Beyotime), staining using crystal violet and counting under a microscope $(100 \times)$.

As for invasion detection, $4 \times 10^{4}$ transfected cells in serum-free medium were plated in the upper chambers pre-coated with Matrigel (BD Biosciences). Remaining steps were consistent with migration assay.

\section{Target Prediction}

Circular RNA Interactome (https://circinteractome.nia.nih. gov/index.html) and TargetScan (http://www.targetscan.org/ mamm_31/) were used to search the target genes of hsa circ_0000144 and miR-502-5p, respectively. And miR-502$5 \mathrm{p}$ and ADAM9 were predicted to be corresponding candidates. 
Table I The Primer Sequences for qPCR in This Study

\begin{tabular}{|c|c|c|}
\hline & Primer & equences $\left(5^{\prime}-3^{\prime}\right)$ \\
\hline \multirow{2}{*}{$\begin{array}{l}\text { has-circ } \\
\text { 0000I44 }\end{array}$} & Forward & GAGTGTTGGCCTGTCCTCAA \\
\hline & Reverse & TTGTGCCCAGTTGCCTGTAT \\
\hline \multirow[t]{2}{*}{ miR-II78 } & Forward & TGCTCACTGTTCTTCCC \\
\hline & Reverse & GAACATGTCTGCGTATCTC \\
\hline \multirow[t]{2}{*}{ miR-I276 } & Forward & AAGAGCCCTGTGGAGAC \\
\hline & Reverse & GAACATGTCTGCGTATCTC \\
\hline \multirow[t]{2}{*}{ miR-197 } & Forward & TTСАССАССТTСТССАС \\
\hline & Reverse & GAACATGTCTGCGTATCTC \\
\hline \multirow[t]{2}{*}{ miR-2I7 } & Forward & TACTGCATCAGGAACTGA \\
\hline & Reverse & GAACATGTCTGCGTATCTC \\
\hline \multirow[t]{2}{*}{ miR-485-3p } & Forward & AGAGGCTGGCCGTGAT \\
\hline & Reverse & GAACATGTCTGCGTATCTC \\
\hline \multirow[t]{2}{*}{$m i R-502-5 p$} & Forward & TCCTTGCTATCTGGGTG \\
\hline & Reverse & GAACATGTCTGCGTATCTC \\
\hline \multirow[t]{2}{*}{ miR-526b } & Forward & CTCTTGAGGGAAGCACT \\
\hline & Reverse & GAACATGTCTGCGTATCTC \\
\hline \multirow[t]{2}{*}{ miR-532-3p } & Forward & GCCTTGAGTGTAGGACC \\
\hline & Reverse & GAACATGTCTGCGTATCTC \\
\hline \multirow[t]{2}{*}{ miR-554 } & Forward & TAGTCCTGACTCAGCCA \\
\hline & Reverse & GAACATGTCTGCGTATCTC \\
\hline \multirow[t]{2}{*}{ miR-580 } & Forward & TTGAGAATGATGAATCATTAG \\
\hline & Reverse & GAACATGTCTGCGTATCTC \\
\hline \multirow[t]{2}{*}{ miR-583 } & Forward & CAAAGAGGAAGGTCCCA \\
\hline & Reverse & GAACATGTCTGCGTATCTC \\
\hline \multirow[t]{2}{*}{ miR-6I0 } & Forward & GAGCTAAATGTGTGCTGG \\
\hline & Reverse & GAACATGTCTGCGTATCTC \\
\hline \multirow[t]{2}{*}{ miR-623 } & Forward & CCTTGCAGGGGCTGTT \\
\hline & Reverse & GAACATGTCTGCGTATCTC \\
\hline \multirow[t]{2}{*}{ miR-942 } & Forward & TCTTCTCTGTTTTGGCCA \\
\hline & Reverse & GAACATGTCTGCGTATCTC \\
\hline \multirow[t]{2}{*}{ ADAM9 } & Forward & GCTAGTTGGACTGGAGATTTGG \\
\hline & Reverse & TTATTACCACAGGAGGGAGCAC \\
\hline \multirow[t]{2}{*}{ MAP7 } & Forward & CAACAAGCGGCTCTCAAAGTCC \\
\hline & Reverse & GCGTCATCAGACGATCCACGAT \\
\hline
\end{tabular}

(Continued)
Table I (Continued)

\begin{tabular}{|l|l|l|}
\hline \multirow{2}{*}{ IRS4 } & \multicolumn{2}{|l|}{ Primer Sequences (5'-3') } \\
\hline \multirow{2}{*}{ NRP2 } & Forward & GACGAGATGCTTTTCACCAGGC \\
\cline { 2 - 3 } & Reverse & GCACTGAAACCGCTCTCCTTGA \\
\hline \multirow{2}{*}{ E2F3 } & Forward & GTCTCCTACAGCCTAAACGGCA \\
\cline { 2 - 3 } & Reverse & GGGTCAAACCTTCGGATGTCAG \\
\hline \multirow{2}{*}{ I8S rRNA } & Forward & AGCGGTCATCAGTACCTCTCAG \\
\cline { 2 - 3 } & Reverse & TGGTGAGCAGACCAAGAGACGT \\
\cline { 2 - 3 } & Reverse & GCCCTCCAATTGTTCCTCGTTAAG \\
\hline \multirow{2}{*}{ U6 snRNA } & Forward & CTCGCTTCGGCAGCACA \\
\cline { 2 - 3 } & Reverse & AACGCTTCACGAATTTGCGT \\
\hline
\end{tabular}

\section{Dual-Luciferase Reporter Assay}

To validate the above prediction, dual-luciferase reporter assay was implemented. The predicted complementary sequences in hsa_circ_0000144 and ADAM9 3'UTR (5'-CAAGGA-3') were mutated into (5'-GUCUCU-3') using GENEART SiteDirected Mutagenesis System (Thermo Fisher Scientific). Then, the wild-type and mutant hsa_circ_0000144 and ADAM9 were cloned into pmirGlO Dual-Luciferase miRNA Target Expression Vector (Promega, Madison, WI, USA) to generate hsa_circ_0000144-wt, hsa_circ_0000144mut, ADAM9-wt and ADAM9-mut. AGS/OXA and MKN45/ OXA cells were co-transfected with above vector and NC or miR-502-5p. Forty-eight hours later, luciferase density was determined by Dual-Glo Luciferase Assay System kit (Promega) in accordance with the user's manual.

\section{RNA Immunoprecipitation (RIP) Assay}

A RIP assay was also conducted to evaluate the direct binding between miR-502-5p and hsa_circ_0000144 or ADAM9 with EZ-Magna RIP Kit (Millipore, Billerica, MA, USA). In short terms, AGS/OXA and MKN45/OXA cells were lysed in specified lysis buffer supplied by kit. Generated cell lysate was mixed with RIP buffer containing magnetic beads conjugated with antibody against Ago2 (ab32381, 1:50 dilution; Abcam, Shanghai, China) or control IgG (ab109489, 1:100 dilution; Abcam). After digestion with Proteinase K (Invitrogen), coprecipitated RNA was analyzed by RT-qPCR assay to detect the enrichment of hsa_circ_0000144, miR-502-5p and ADAM9. 


\section{Western Blot Analysis}

Protein samples were prepared using RadioImmunoprecipitation Assay (RIPA; Beyotime), followed by concentration measurement using a bicinchoninic acid protein assay kit (Thermo Fisher Scientific). Subsequently, $40 \mu \mathrm{g}$ protein samples were separated by fresh sodium dodecyl sulfate-polyacrylamide gel electrophoresis (SDSPAGE) and transferred onto the polyvinylidene fluoride membranes (Millipore). After blockage with 5\% defatted milk, membranes were incubated with primary antibody against ADAM9 (ab218242, 1: 2000 dilution; Abcam), proliferating cell nuclear antigen (PCNA; ab92552, 1:2000 dilution; Abcam), ATP binding cassette subfamily B member 1 (ABCB1; ab235954, 1:3000 dilution; Abcam) or loading control $\beta$-actin (ab124964, 1:5000 dilution; Abcam) at $4^{\circ} \mathrm{C}$ overnight, and then probed with secondary antibody (ab205718, 1:10,000 dilution; Abcam) at room temperature for $1 \mathrm{~h}$. At last, protein signals were activated exploiting enhanced chemiluminescence kit (Thermo Fisher Scientific) and evaluated using Image J software.

\section{Xenograft Tumor Assay}

This assay was performed under the ratification from the Ethics Committee of Baoji Central Hospital. Lentivirus vectors embracing harboring short hairpin RNA (shRNA) targeting hsa_circ_0000144 (Lenti-sh-hsa_circ_0000144) and its negative control (Lenti-sh-NC) were constructed by Hanbio Biotechnology Co., ltd (Shanghai, China). BALB/c nude mice (male, 5 weeks old) bought from Shanghai SLAC Laboratory Animal Co., Ltd. (Shanghai, China) were subcutaneously injected with AGS/OXA cells $\left(2 \times 10^{6}\right)$ stably transfected with Lenti-sh-hsa_circ_0000144 or Lenti-sh-NC $(\mathrm{n}=5)$. After $5 \mathrm{~d}$, volume of generated tumors was recorded (Volume $=$ length $\times$ width $/ 2$ ) every $5 \mathrm{~d}$. And each mouse was intraperitoneally inoculated with OXA $(35 \mathrm{mg} / \mathrm{kg})$ every 5 $\mathrm{d}$ for 5 times. Thirty days later, all mice were killed, and tumor volume inhibition rate was calculated. And tumor tissues were collected and weighed, followed by RT-qPCR and Western blot assays.

\section{Statistical Analysis}

All data were generated from $\geq 3$ independent experiments, and analyzed by SPSS 21.0 (SPSS, Chicago, IL, USA), then expressed as mean \pm standard deviation. Difference was assessed by Student $t$-test or one-way analysis of variance (ANOVA) followed by Tukey's test. $P<0.05$ was considered as statistically significant. Moreover, correlation analysis among hsa circ 0000144, miR-502-5p and ADAM9 in GC tissues was conducted with Pearson's correlation analysis.

\section{Results}

Hsa_circ_0000I44 Expression Was Upregulated in OXA-Resistant GC Tissues and Cells

According to microarray data GSE78092 downloaded from the Gene Expression Omnibus (GEO) database, hsa_circ_0000144 was highly enriched in GC tissues (Figure 1A). We then detected the relative expression level of hsa_circ_0000144 in 25 cases of OXA-sensitive and 25 cases of OXA-resistant GC tissues. As exhibited in Figure 1B, hsa_circ_0000144 was upregulated in both OXA-sensitive and OXA-resistant GC tissues when compared to normal tissues, especially in OXA-resistant GC tissues. Likewise, we found that hsa_circ_0000144 expression was higher in AGS and MKN45 cells relative to GES1 cells. Additionally, hsa_circ_0000144 was upregulated in OXA-resistant GC cells (AGS/OXA and MKN45/OXA cells) in contrast to corresponding parental OXA-sensitive GC cells (Figure 1C). In order to explore the potential molecular mechanism of hsa_circ_0000144 in GC development, the subcellular location was determined via nuclear/cytoplasmic fractionation. Results showed that hsa_circ_0000144 was mainly located in the cytoplasm of AGS/OXA and MKN45/OXA cells (Figure 1D). Moreover, the expression level of linear mRNA, rather than hsa_circ_0000144, was declined in AGS/OXA and MKN45/OXA cells after RNase R digestion, suggesting the stable structure of hsa_circ_0000144 (Figure 1E). Taken together, hsa_circ_0000144 was upregulated in OXA-resistant GC tissues and cells.

\section{Hsa_circ_0000 I44 Depletion Suppressed OXA Resistance, Proliferation and Metastasis in OXA-Resistant GC Cells}

CCK-8 assay was carried out to analyze the survival rate of AGS/OXA and MKN45/OXA cells as well as corresponding parental OXA-sensitive GC cells treated by OXA at different concentrations. As illustrated in Figure 2A, OXA treatment efficiently reduced the survival rate of cells in a concentration-dependent way. The IC50 values of OXA in AGS, AGS/OXA, MKN45 and MKN45/OXA cells were 60.98, 212.8, 70.57 and 289.2, indicating AGS/OXA and MKN45/OXA were 
A



B

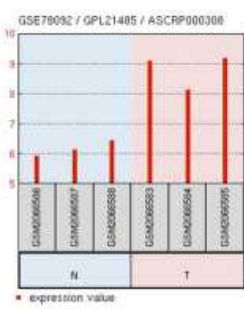

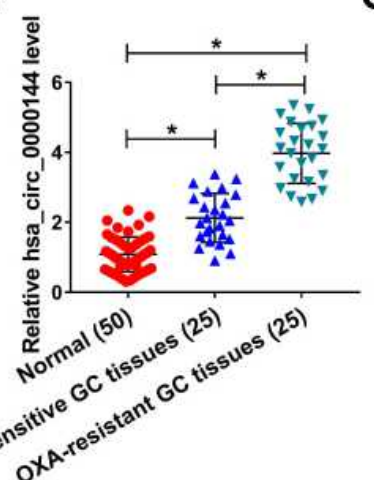

C

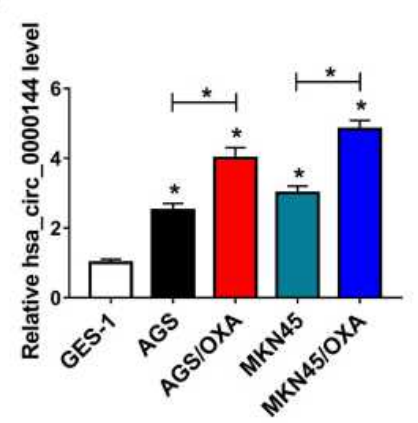

D

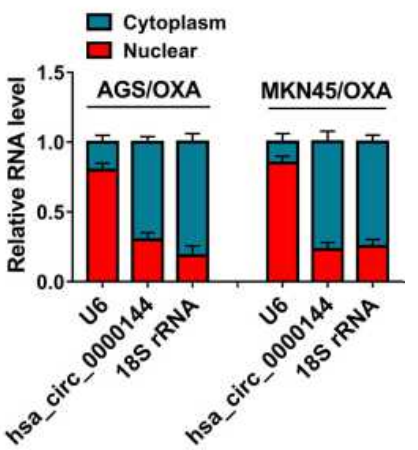

Figure I Hsa_circ_0000I44 expression was upregulated in OXA-resistant GC tissues and cells. (A) Expression of hsa_circ_0000I44 in GC tissues and normal tissues showed by GSE78092 database. (B) RT-qPCR assay for the expression of hsa_circ_0000I44 in normal tissues, 25 cases of OXA-sensitive and 25 cases of OXA-resistant GC tissues. (C) RT-qPCR assay for the expression of hsa_circ_0000I44 in GES-I, AGS, AGS/OXA, MKN45 and MKN45/OXA cells. (D) Nuclear-cytoplasmic fractionation for the location of hsa_circ_0000I44 in AGS/OXA and MKN45/OXA cells. (E) RT-qPCR assay for the expression of hsa_circ_0000I44 and linear mRNA in RNA isolated from AGS/OXA and MKN45/OXA cells digested with RNase $\mathrm{R}$ or not. $* P<0.05$.

OXA-resistant cell lines. Then, siRNAs against hsa_circ_0000144 (si-hsa_circ_0000144\#1, si-hsa _circ_0000144\#2 and si-hsa_circ_0000144\#3) and siNC were introduced into AGS/OXA and MKN45/ OXA cells. Among the three, si-hsa_circ_0000144\#1 exhibited the best knockdown efficiency (Figure 2B). Therefore, it was selected for subsequent assays. Hsa_circ_0000144 knockdown distinctly reduced the IC50 values of OXA in AGS/OXA (from 222.5 to 88.49) and MKN45/OXA cells (from 295.1 to 99.09) (Figure 2C). Colony formation assay revealed that hsa_circ_0000144 depletion also inhibited the colony formation ability of OXA-resistant GC cells (Figure 2D). Moreover, silencing of hsa_circ_0000144 obviously elevated apoptotic rate (Figure 2E) and caspase 3 activity (Figure 2F) in AGS/OXA and MKN45/ OXA cells. Transwell assay showed that hsa_circ_0000144 depletion also repressed migration and invasion in OXA-resistant GC cells (Figure 2G and H). Moreover, depletion of hsa_circ_0000144 efficiently reduced the protein level of chemoresistancerelated protein ABCB1 in OXA-resistant GC cells. (Supplementary Figure S1C). Taken together, hsa_circ_0000144 knockdown inhibited OXA resistance, proliferation and metastasis in OXAresistant GC cells.

\section{MiR-502-5p Was a Direct Target of hsa_circ_0000I44}

Mounting has suggested that circRNAs could sponge miRNAs to regulate the stability and transcription of mRNAs in cancer. $^{23}$ Herein, circular RNA Interactome predicted that there existed 14 possible target miRNAs of hsa_circ_0000144, including miR-1178, miR-1276, miR197, miR-217, miR-485-3p, miR-502-5p, miR-526b, miR-532-3p, miR-554, miR-580, miR-583, miR-610, miR-623 and miR-942. Additionally, the expression level of miR-502-5p in OXA-resistant GC cells with hsa_circ_0000144 overexpression exhibited the most significant downregulation among these 14 miRNAs (Supplementary Figure S1A), thus miR-502-5p was chosen for further investigation. The binding sites between hsa_circ_0000144 and miR-502-5p are shown in Figure 3A. Dual-luciferase reporter assay revealed that transfection with miR-502-5p, other than NC, significantly repressed the luciferase density of AGS/OXA and MKN45/OXA cells co-transfected with hsa_circ_0000144-wt, resulting in about $70 \%$ reduction. 


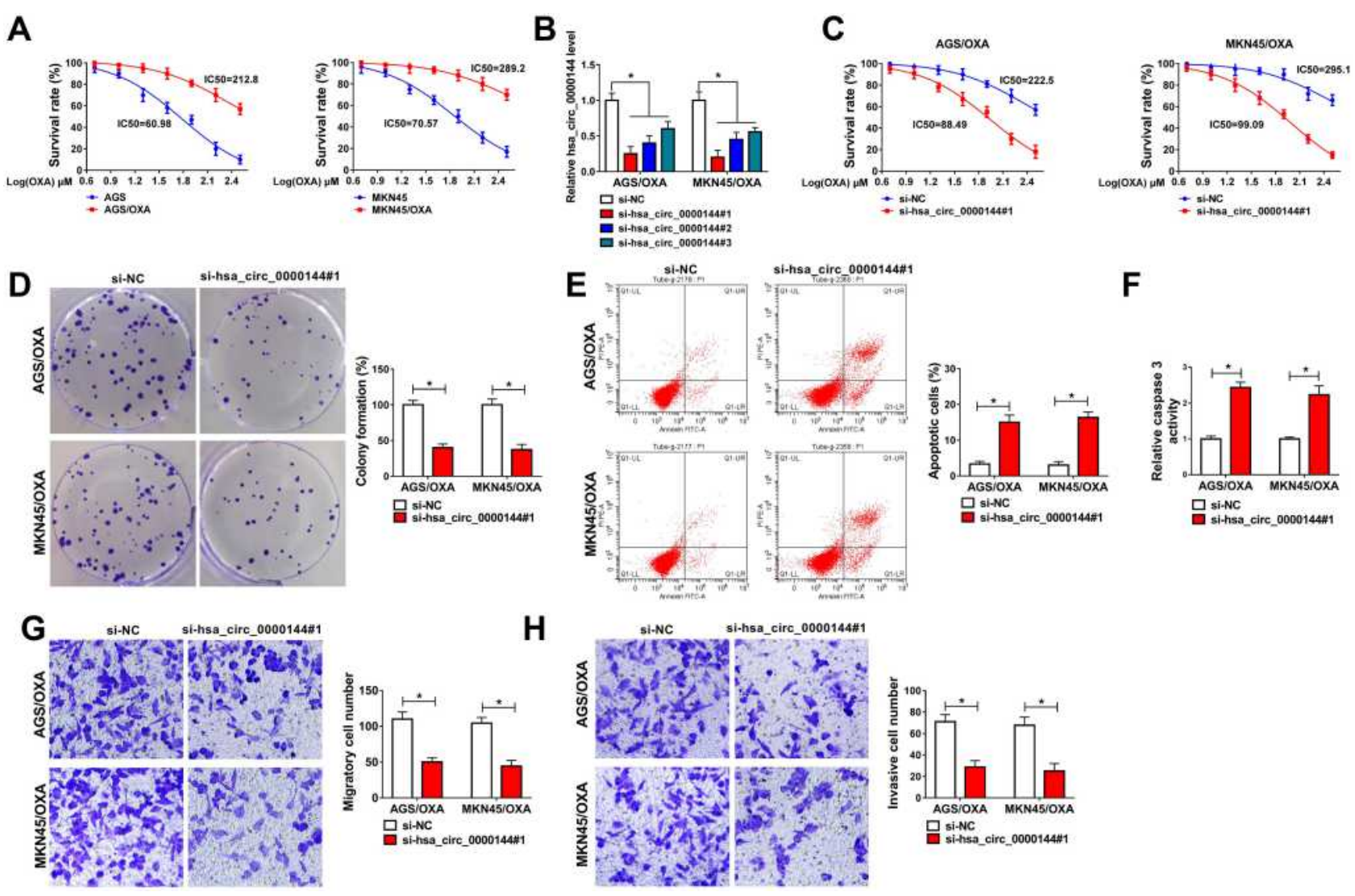

Figure 2 Hsa_circ_0000I44 depletion suppressed OXA resistance, proliferation and metastasis in OXA-resistant GC cells. (A) CCK-8 assay for the survival rate of AGS, AGS/OXA, MKN45 and MKN45/OXA cells treated with OXA at different concentrations (5, 10, 20, 40, 80,160 or $320 \mu M)$. (B) RT-qPCR assay for the expression of hsa_circ_0000I44 in AGS/OXA and MKN45/OXA cells transfected with si-NC, si-hsa_circ_0000I44\#I, si-hsa_circ_0000I44\#2 or si-hsa_circ_0000I44\#3. (C-H) AGS/ OXA and MKN45/OXA cells were transfected with si-NC or si-hsa_circ_0000I44\#I. (C) CCK-8 assay for the survival rate of transfected cells treated with OXA at different concentrations (5, 10, 20, 40, 80, 160 or $320 \mu$ M). (D) Colony formation assay for the colony formation ability of transfected cells. (E) Flow cytometry for the cell apoptosis in transfected cells. (F) Caspase 3 activity in transfected cells determined by commercial kit. (G and $\mathbf{H})$ Transwell assay for the cell migration and invasion in transfected cells. $* P<0.05$.

While luciferase activity of cells co-transfected with hsa_circ_0000144-mut changed little (Figure 3B). RIP assay showed that both hsa_circ_0000144 and miR-502$5 p$ were highly enriched in AGS/OXA and MKN45/OXA cells in Anti-Ago2 group (Figure 3C). As we could see in Figure 3D, hsa_circ_0000144 expression was apparently increased in AGS/OXA and MKN45/OXA cells due to transfection with hsa_circ_0000144 overexpression plasmid, and cells introduced with circ-NC acted as control. Then, RT-qPCR assay proved the hsa_circ_0000144 overexpression-induced downregulation of miR-502-5p and hsa_circ_0000144 depletion-induced upregulation of miR-502-5p in AGS/OXA and MKN45/OXA cells
(Figure 3E). As expected, miR-502-5p expression was downregulated in GC tissues in contrast to normal tissues, especially in OXA-resistant GC tissues (Figure 3F). Furthermore, hsa_circ_0000144 expression in GC tissues was inversely correlated with that of miR-502-5p $(P<$ $0.0001, r=-0.7863$ ) (Figure 3G). Similarly, miR-502-5p was downregulated in OXA-sensitive and OXA-resistant GC cells when compared with GES-1 cells. And expression level of miR-502-5p in AGS/OXA and MKN45/ OXA cells was lower than that in AGS and MKN45 cells, respectively (Figure $3 \mathrm{H}$ ). The above results suggested that hsa_circ_0000144 could target miR-502-5p and negatively regulated its expression. 
A

hsa_circ_0000144-mut $\left(5^{\prime} \ldots 3^{\prime}\right)$
hsa-miR-502-5p $\left(3^{\prime} \ldots 5^{\prime}\right)$

hsa_circ_0000144-mut $\left(5^{\prime} . . .3^{\prime}\right)$

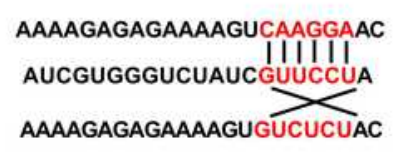

B


C
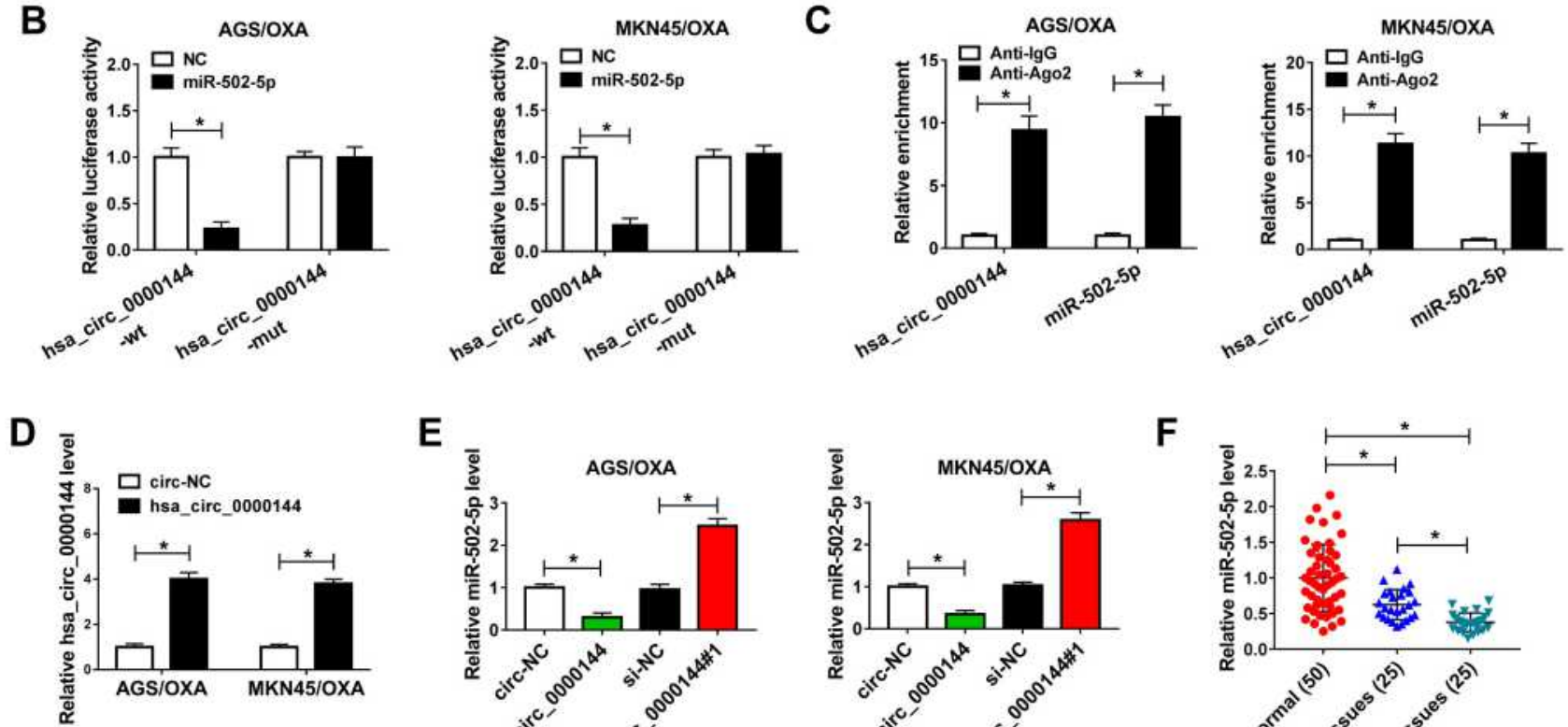

E
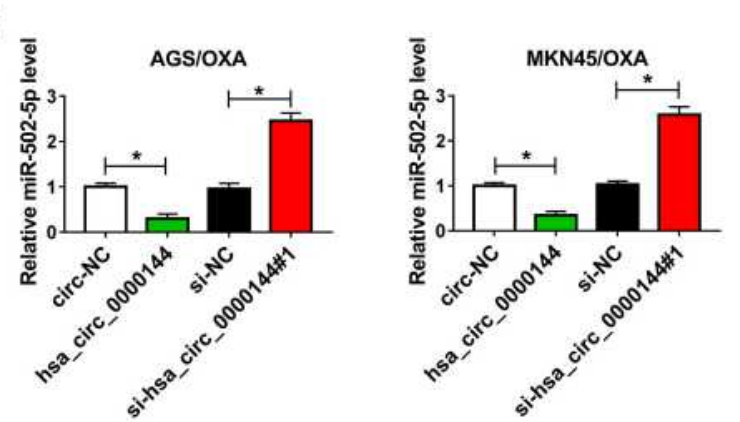

F

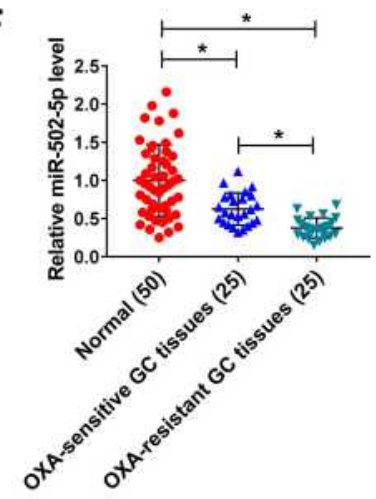

G

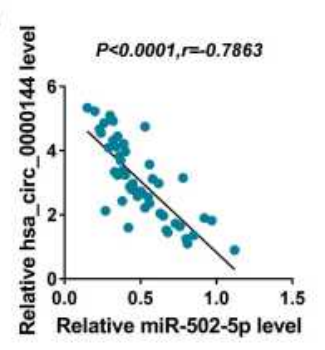

H

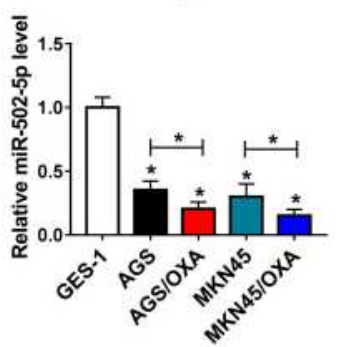

Figure 3 MiR-502-5p was a direct target of hsa_circ_0000I44. (A) The predicted binding positions between hsa_circ_0000I44 and miR-502-5p by Circular RNA Interactome. (B) Dual-luciferase reporter assay for the luciferase activity of AGS/OXA and MKN45/OXA cells co-transfected with hsa_circ_0000I44-wt or hsa_circ_0000I44-mut and miR-502-5p or NC. (C) RIP and RT-qPCR assays for the binding efficiency of hsa_circ_0000I44 and miR-502-5p to Ago2 protein in AGS/OXA and MKN45/OXA cells. (D) RT-qPCR assay for the expression of hsa_circ_0000I44 in AGS/OXA and MKN45/OXA cells transfected with circ-NC or hsa_circ_0000I44. (E) RT-qPCR assay for the expression of miR-502-5p in AGS/OXA and MKN45/OXA cells transfected with circ-NC or hsa_circ_0000I44. (F) RT-qPCR assay for the expression of miR-502-5P in normal tissues, 25 cases of OXA-sensitive and 25 cases of OXA-resistant GC tissues. (G) Pearson's correlation analysis for the correlation between hsa circ 0000144 and miR-502-5p in 50 cases of GC tissues. (H) RT-qPCR assay for the expression of miR-502-5p in GES-I, AGS, AGS/OXA, MKN45 and MKN45/OXA cells. $* p<0.05$.

\section{MiR-502-5p Inhibition Weakened hsa_circ_0000I44 Depletion-Mediated Inhibitory Effects on OXA Resistance, Proliferation and Metastasis in OXA-Resistant GC Cells}

We then investigated the role of miR-502-5p in hsa_circ_0000144 knockdown-mediated OXA-resistant GC cells. After transfection with miR-502-5p inhibitor, miR- 502-5p expression was remarkably decreased in AGS/ OXA and MKN45/OXA cells (Figure 4A). And miR502-5p inhibition almost reversed hsa_circ_0000144 knockdown-induced the upregulation of miR-502-5p in AGS/OXA and MKN45/OXA cells (Figure 4B). Furthermore, aforementioned declined IC50 values of OXA (Figure 4C), reduced colony formation ability (Figure 4D), elevated apoptotic rate (Figure 4E) and caspase 3 activity (Figure 4F), as well as inhibited migration 
A

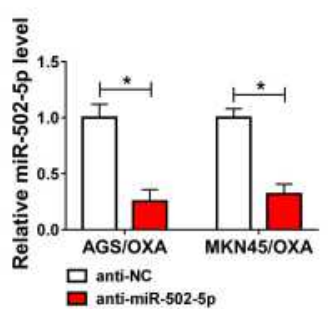

D

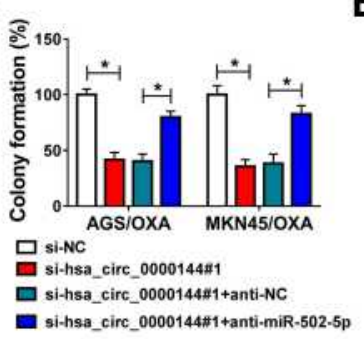

B

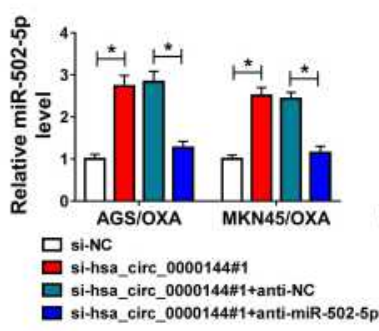

C

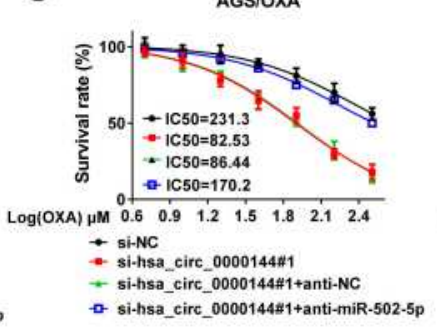



$\mathbf{F}$



$\square$ si-NC

口i-hsa_circ_0000144\#t

口i-hsa_circ_0000144\#1+anti-NC

- si-hsa_circ_0000144\#1+anti-miR-502-5p

G
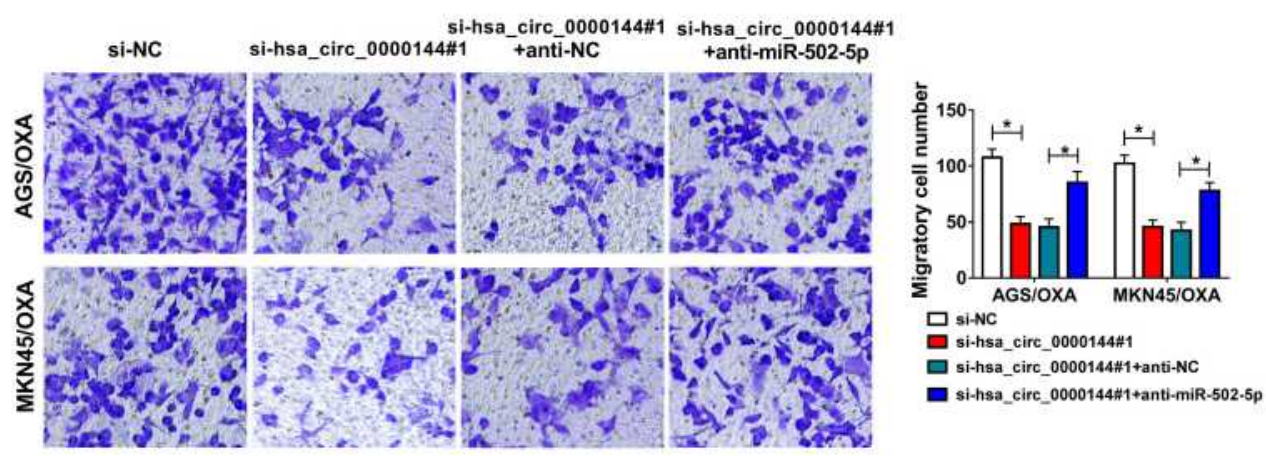

H
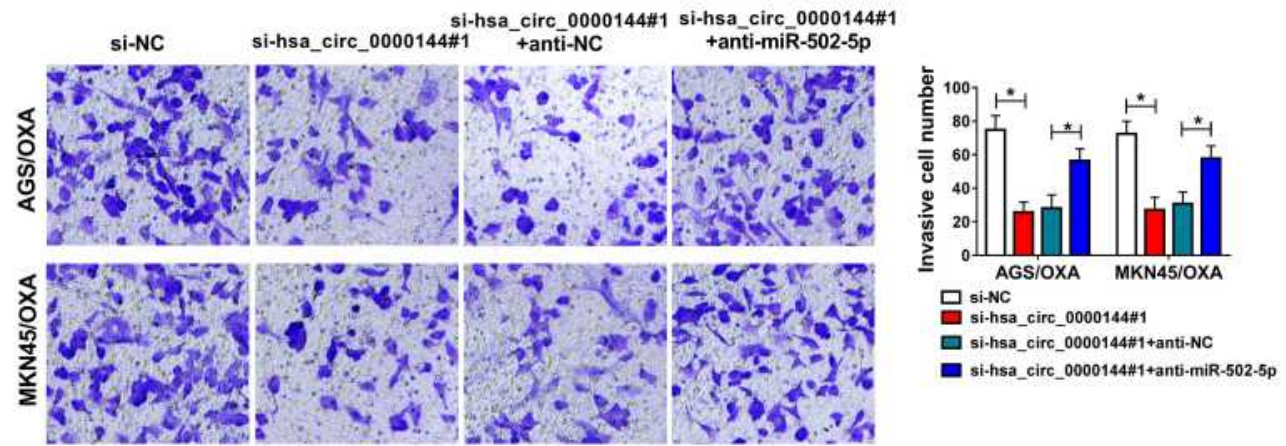

Figure 4 MiR-502-5p inhibition weakened hsa_circ_0000I44 depletion-mediated inhibitory effects on OXA resistance, proliferation and metastasis in OXA-resistant GC cells. (A) RT-qPCR assay for the expression of miR-502-5p in AGS/OXA and MKN45/OXA cells transfected with anti-NC or anti-miR-502-5p. (B-H) AGS/OXA and MKN45/OXA cells were transfected with si-NC, si-hsa_circ_0000I44\#I si-hsa_circ_0000I44\#I+anti-NC or si-hsa_circ_0000I44\#I+anti-miR-502-5p. (B) RT-qPCR assay for the expression of miR-502-5p in transfected cells. (C) CCK-8 assay for the survival rate of transfected cells treated with OXA at different concentrations $(5,10,20,40$, 80,160 or $320 \mu \mathrm{M}$ ). (D) Colony formation assay for the colony formation ability of transfected cells. (E) Flow cytometry for the cell apoptosis in transfected cells. (F) Caspase 3 activity in transfected cells determined by commercial kit. ( $\mathbf{G}$ and $\mathbf{H}$ ) Transwell assay for the cell migration and invasion in transfected cells. $* P<0.05$.

and invasion (Figure $4 \mathrm{G}$ and $\mathrm{H}$ ) in AGS/OXA and MKN45/OXA cells caused by hsa_circ_0000144 depletion were all largely relieved by interference of miR-502-5p. Therefore, hsa_circ_0000144 deficiency repressed OXA resistance, proliferation and metastasis in OXA-resistant GC cells by antagonizing miR-502-5p.

\section{MiR-502-5p Could Directly Target ADAM9}

CircRNAs have been reported to function in tumorigenesis and development of GC by serving as competing endogenous RNAs (ceRNAs) of miRNAs to modulate the expression of target mRNAs. ${ }^{24}$ Here, TargetScan 
A
ADAM9-wt 3 ' UTR
5'...GAGAGUUGUGAAAUACAAGGAAA... 3'
hsa-miR-502-5p
ADAM9-mut 3 ' UTR

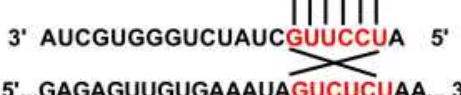

B
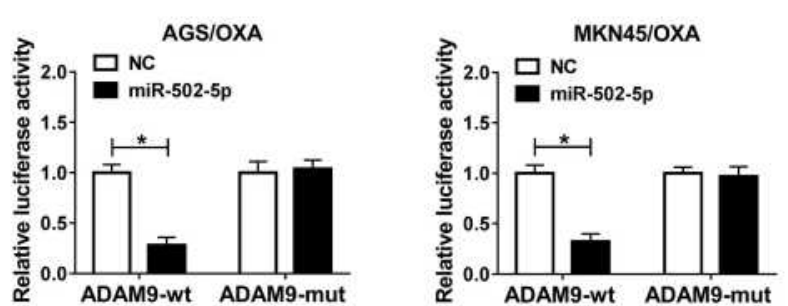

C
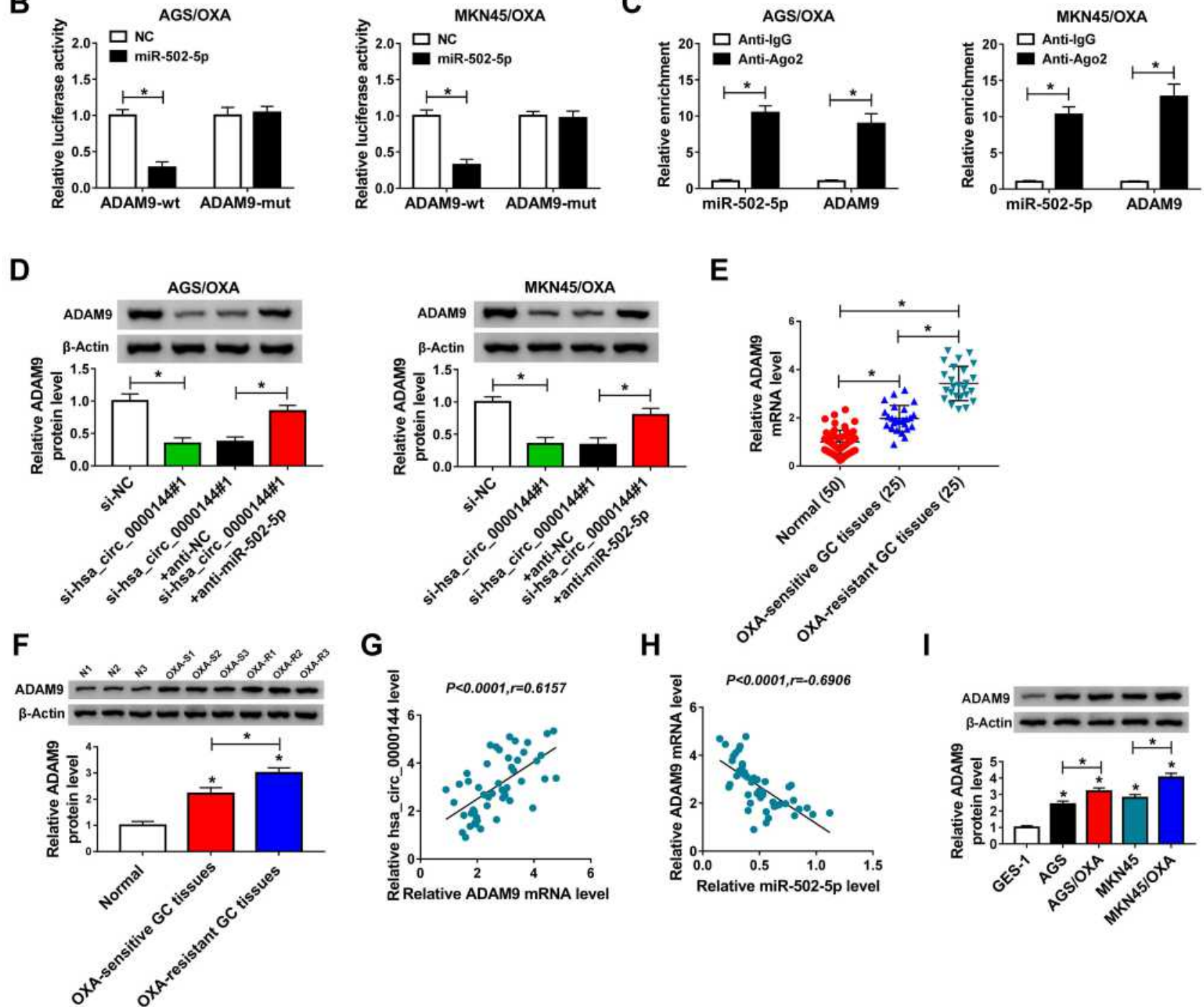

$\mathbf{E}$

G

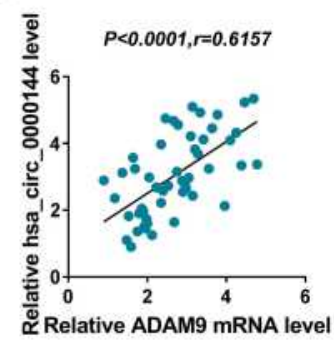

H
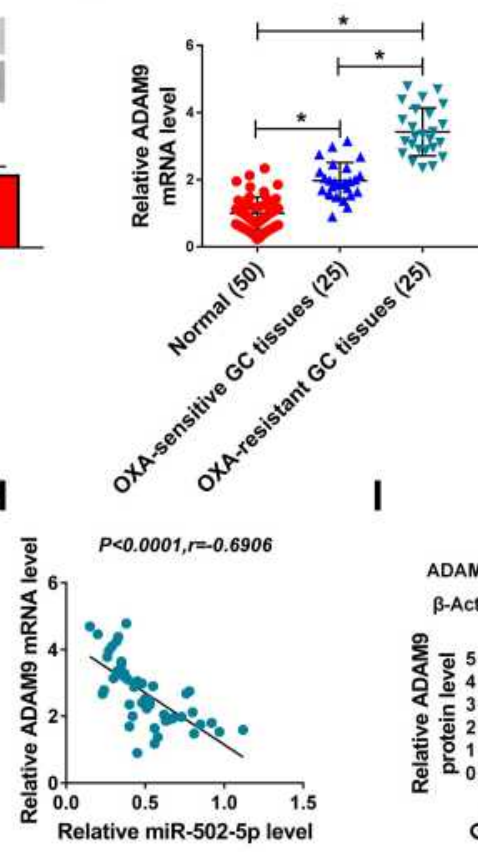

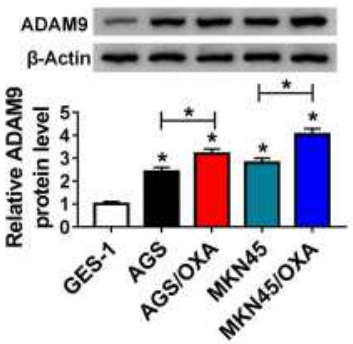

Figure 5 MiR-502-5p could directly target ADAM9. (A) The predicted binding sites between miR-502-5p and ADAM9 3'UTR by TargetScan. (B) Dual-luciferase reporter assay for the luciferase activity of AGS/OXA and MKN45/OXA cells co-transfected with ADAM9-wt or ADAM9-mut and miR-502-5p or NC. (C) RIP and RT-qPCR assays for the binding efficiency of miR-502-5p and ADAM9 to Ago2 protein in AGS/OXA and MKN45/OXA cells. (D) Western blot assay for the protein level of ADAM9 in AGS/ OXA and MKN45/OXA cells transfected with si-NC, si-hsa_circ_0000I44\#I, si-hsa_circ_0000I44\#I+anti-NC or si-hsa_circ_0000I44\#I+anti-miR-502-5p. (E) RT-qPCR assay for the mRNA expression of ADAM9 in normal tissues, 25 cases of OXA-sensitive and 25 cases of OXA-resistant GC tissues. (F) Western blot assay for the protein level of ADAM9 in normal tissues, OXA-sensitive and OXA-resistant GC tissues. (G) Pearson's correlation analysis for the correlation between hsa_circ_0000I44 and ADAM9 mRNA in 50 cases of GC tissues. (H) Pearson's correlation analysis for the correlation between miR-502-5p and ADAM9 mRNA in 50 cases of GC tissues. (I) RTqPCR assay for the mRNA expression of ADAM9 in GES-I, AGS, AGS/OXA, MKN45 and MKN45/OXA cells. *P < 0.05.

was utilized to predict the downstream gene of miR502-5p. As shown in Supplementary Figure S1B, ADAM9, MAP7, IRS4, NRP2 and E2F3 mRNA were predicted that contained the complementary binding sites for miR-502-5p. Besides, the levels of target mRNAs, especially ADAM9, were dramatically declined in AGS/OXA cells transfected with miR-502-5p mimic
(Supplementary Figure S1B). To verify the predictions, ADAM9 3'UTR sequences harboring wild type (wt) or mutant type (mut) miR-502-5p binding sites were inserted into the luciferase reporter plasmids (Figure $5 \mathrm{~A})$. Introduction of miR-502-5p efficiently decreased the luciferase density of AGS/OXA and MKN45/OXA cells co-transfected with ADAM9-wt relative to $\mathrm{NC}$. 
The luciferase density of cells co-transfected with ADAM9-mut was changeless (Figure 4B). Following RIP assay also confirmed the binding potency between miR-502-5p and ADAM9 (Figure 5C). As demonstrated via Western blot assay, hsa_circ_0000144 knockdown downregulated the protein level of ADAM9 in AGS/ OXA and MKN45/OXA cells, which was attenuated by miR-502-5p inhibitor (Figure 5D). The mRNA (Figure 5E) and protein (Figure 5F) levels of ADAM9 were upregulated in GC tissues in comparison with normal tissues, especially in OXA-resistant GC tissues. There existed a positive correlation between hsa_circ_0000144 expression and ADAM9 mRNA expression $(P<0.0001, r=0.6157)$ (Figure 5G), as well as a negative correlation between miR-502-5p expression and ADAM9 mRNA expression in GC tissues $(P<$ 0.0001, $r=-0.6906$ ) (Figure 5H). As illustrated in Figure 5I, ADAM9 protein level was upregulated in GC cells. When compared to OXA-sensitive GC cells, ADAM9 protein level was higher in OXA-resistant GC cells. To sum up, miR-502-5p targeted ADAM9 in OXA-resistant GC cells.

\section{Overexpression of miR-502-5p Reduced OXA Resistance, Proliferation and Metastasis in OXA-Resistant GC Cells by Downregulating ADAM9 Expression}

Subsequently, we further explored the co-effects of miR-502$5 p$ and ADAM9 on cellular behaviors of OXA-resistant GC cells. MiR-502-5p expression apparently elevated in AGS/ OXA and MKN45/OXA cells transfected with miR-502-5p in contrast to cells transfected with NC (Figure 6A). Western bolt assay demonstrated that transfection with pcDNAADAM9 increased ADAM9 protein level in OXA-resistant GC cells compared to pcDNA-NC (Figure 6B). Moreover, overexpression of ADAM9 almost recovered miR-502-5pmediated downregulated protein level of ADAM9 in AGS/ OXA and MKN45/OXA cells (Figure 6C). Furthermore, miR-502-5p-mediated decreased IC50 values of OXA (Figure 6D and E), inhibited colony formation ability (Figure 6F), raised apoptotic rate (Figure 6G) and caspase 3 activity (Figure $6 \mathrm{H}$ ), as well as repressed migration and invasion (Figure 6I and J) were all largely rescued by upregulation of ADAM9. Therefore, miR-502-5p exerted repressed impact on OXA resistance, proliferation and metastasis in OXA-resistant GC cells by downregulating ADAM9 expression.

\section{Hsa_circ_0000I44 Depletion Inhibited GC Tumor Growth in vivo}

AGS/OXA cells stably expressing sh-hsa_circ_0000144 or sh-NC were injected into nude mice, followed by inoculation with OXA. As shown in Figure 7A and B, hsa_circ_0000144 knockdown reduced tumor growth, including volume and weight, and hsa_circ_0000144 depletion induced higher tumor volume inhibition rate in contrast with sh-NC group. In addition, tumors in Lenti-sh-hsa_circ_0000144 or Lenti-sh-hsa _circ_0000144+OXA group exhibited lower expression of hsa_circ_0000144, ADAM9 and PCNA (proliferation biomarker), as well as higher expression of miR-502-5p relative to corresponding control (Figure 7C and D). The above data manifested that hsa_circ_0000144 knockdown could block GC tumor growth in vivo.

\section{Discussion}

CircRNAs were identified as promising diagnostic biomarkers and therapeutic targets of GC. Moreover, the roles of circRNA-miRNA-mRNA axes in GC progression were manifested. $^{25}$ In this project, hsa_circ_0000144 was discovered to be upregulated in OXA-resistant GC tissues and cells. Functionally, silencing of hsa_circ_0000144 had inhibitory effects on OXA resistance, proliferation, metastasis and tumorigenesis in OXA-resistant GC cells, which were attributed to miR-502-5p/ADAM9 axis, at least in part.

As previously reported, hsa_circ_0000144 could facilitate bladder cancer progression by antagonizing miR-217 to upregulate RUNX2 expression, serving as a tumor-promoting agent. $^{26}$ Also known as circSLAMF6, hsa_circ_0000144 brought promoted impact on GC cell proliferation and mobility, with potential applicability for GC diagnosis and treatment. ${ }^{11}$ Fang et al verified that hsa_circ_0000144 expression was upregulated in GC cells under hypoxia conditions. Hsa_circ_0000144 deficiency suppressed glycolysis, metastasis and tumorigenesis of GC cells under hypoxia through the regulation of miR-204-5p/ MYH9 axis. $^{27}$ In addition, hsa_circ_0000144 sponged miR-623 to upregulate GPRC5A expression, so as to promote GC progression. ${ }^{28}$ In keeping with former discoveries, we also detected the upregulation of hsa_circ_0000144 in GC tissues and cells, especially in OXA-resistant tissues and cells. The inhibitory influence of hsa_circ_0000144 knockdown on OXA resistance, 
A

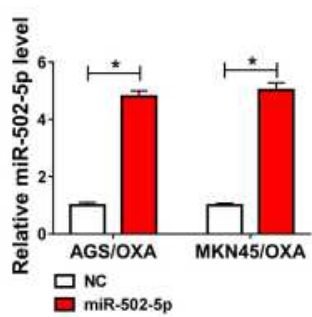

E

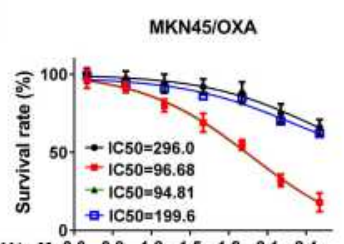

Log(OXA) $\mu \mathrm{M} \begin{array}{llllllll}0.6 & 0.9 & 1.2 & 1.5 & 1.8 & 2.1 & 2.4\end{array}$

$\rightarrow$ NC

- miR-502-5p+pcDNA-NC

- miR-502-5p+pcDNA-ADAM9
B

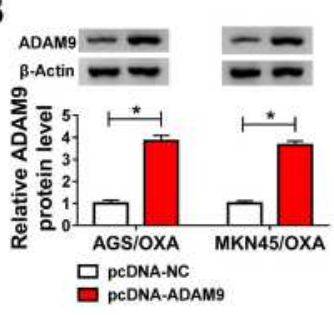

F

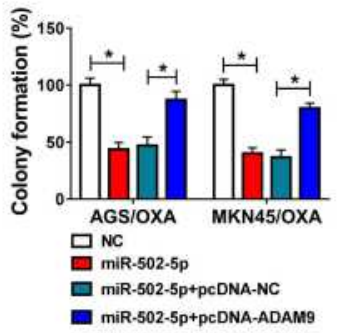

C

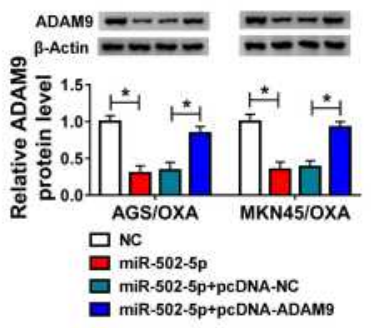

G



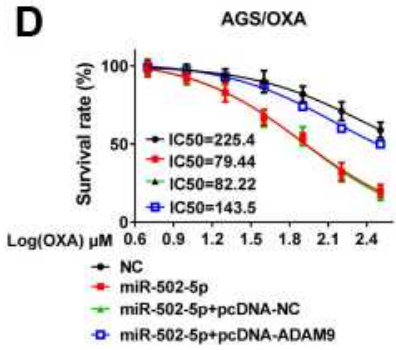

H

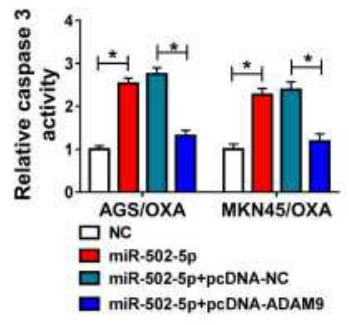

I
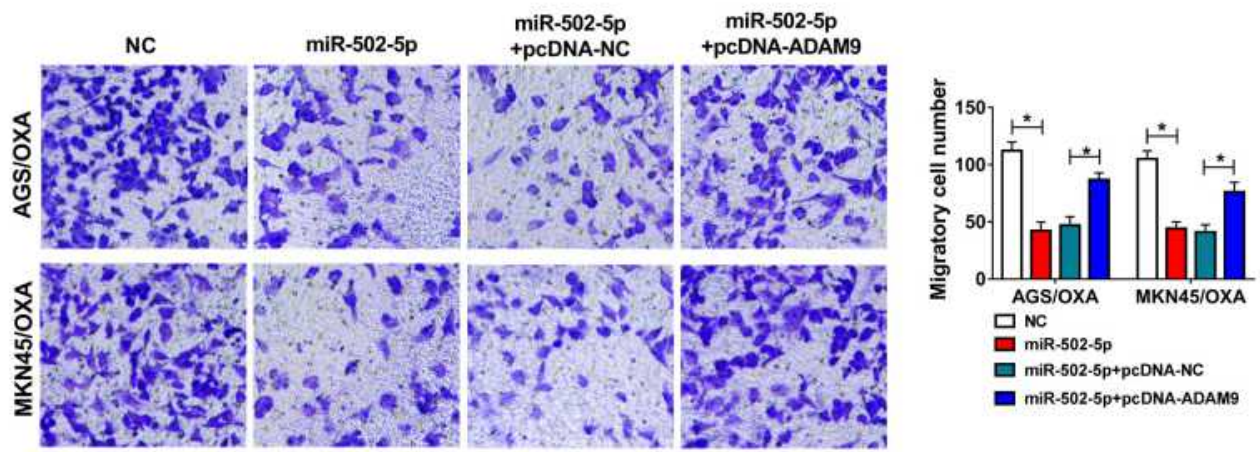

J
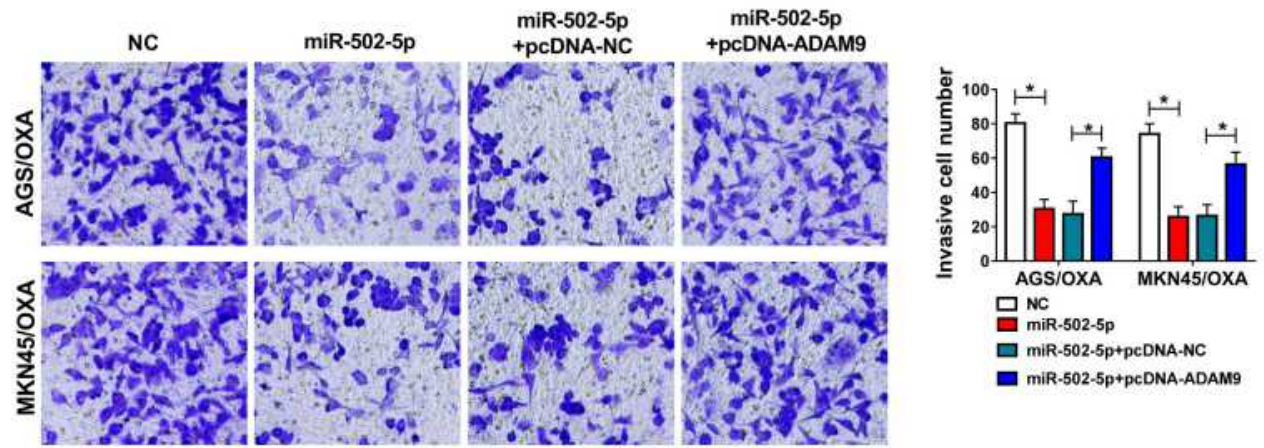

Figure 6 Enforced expression of miR-502-5p reduced OXA resistance, proliferation and metastasis in OXA-resistant GC cells by downregulating ADAM9 expression. (A) RT-qPCR assay for the expression of miR-502-5p in AGS/OXA and MKN45/OXA cells transfected with NC or miR-502-5p. (B) Western blot assay for the protein level of ADAM9 in AGS/OXA and MKN45/OXA cells transfected with pcDNA-NC or pcDNA-ADAM9. (C-J) AGS/OXA and MKN45/OXA cells were transfected with NC, miR502-5p, miR-502-5p+pcDNA-NC or miR-502-5p+pcDNA-ADAM9. (C) Western blot assay for the protein level of ADAM9 in transfected cells. (D and E) CCK-8 assay for the survival rate of transfected cells treated with OXA at different concentrations $(5,10,20,40,80,160$ or $320 \mu \mathrm{M})$. (F) Colony formation assay for the colony formation ability of transfected cells. (G) Flow cytometry for the cell apoptosis in transfected cells. (H) Caspase 3 activity in transfected cells determined by commercial kit. (I and $\mathbf{J})$ Transwell assay for the cell migration and invasion in transfected cells. $* P<0.05$.

proliferation and metastasis in OXA-resistant GC cells in vitro, as well as on tumor growth in vivo was disclosed.
It is widely accepted that circRNAs could function by acting as sponges of target miRNAs. ${ }^{29}$ Herein, miR-502-5p was confirmed as a target miRNA of hsa_circ_0000144. 
A

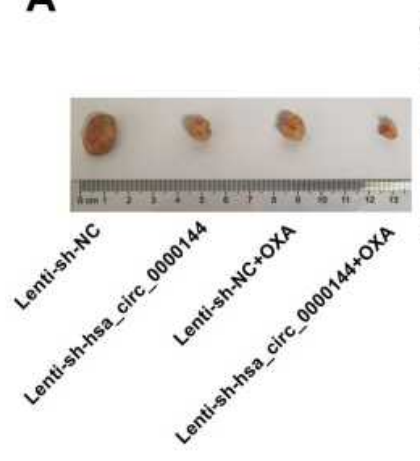

C

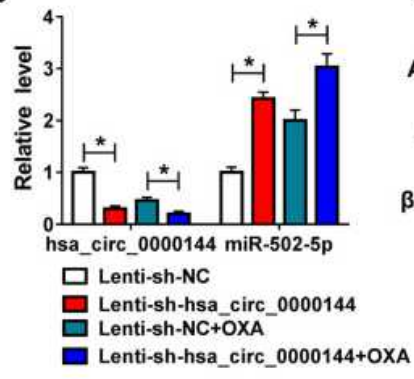

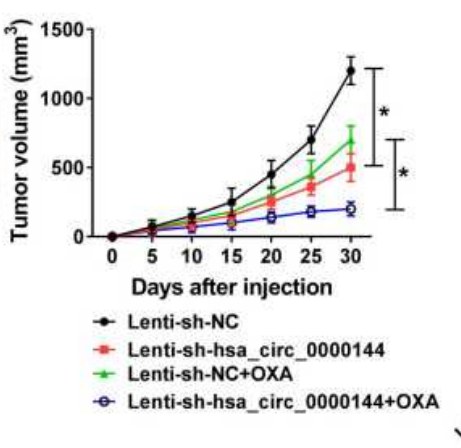

D



B
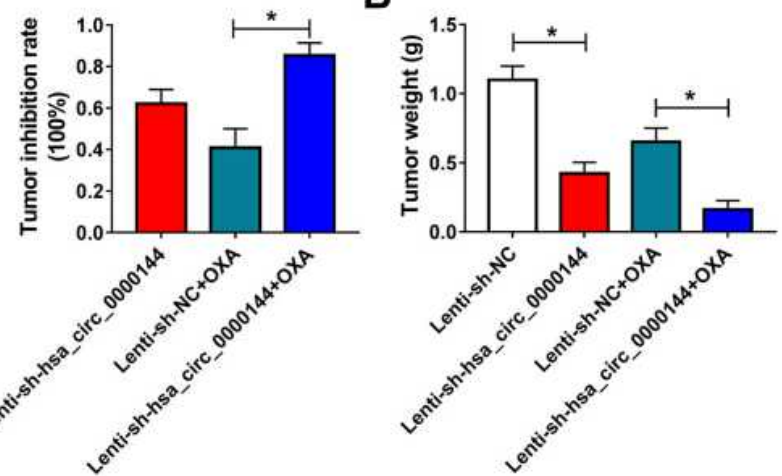

Figure $7 \mathrm{Hsa}$ _circ_0000I44 depletion inhibited GC tumor growth in vivo. AGS/OXA cells stably expressing sh-hsa_circ_0000I44 or sh-NC were injected into nude mice, followed by inoculation with OXA. (A) Tumor volume measured every $5 \mathrm{~d}$, image collected at $30 \mathrm{~d}$ post injection. (B) Tumor weight measured at $30 \mathrm{~d}$ post injection. (C) RTqPCR assay for the expression of hsa_circ_0000I44 and miR-502-5p in generated tumors. (D) Western blot assay for the protein levels of ADAM9 and PCNA generated tumors. $* P<0.05$.

MiR-502-5p was demonstrated to be a tumor suppressor in colon cancer, ${ }^{30}$ breast cancer ${ }^{31}$ and bladder cancer, ${ }^{32}$ as well as in GC. ${ }^{33}$ Peng et al found that miR-502-5p was low expressed in GC. And it exerted anti-proliferation, antimigration and anti-invasion roles in GC cells by downregulating SP1 expression. ${ }^{33}$ Furthermore, miR-502-5p was sponged by circ_0032627, involved in circ_0032627mediated GC progression. ${ }^{34}$ From our data, miR-502-5p was downregulated in GC tissues and cells, as well as in OXA-resistant tissues and cells, similar to above reports. In addition, miR-502-5p efficiently repressed OXA resistance, proliferation and metastasis in OXA-resistant GC cells, performing as a tumor-suppressor role. And miR-502-5p inhibition attenuated hsa_circ_0000144 knockdowninduced repressed impact on OXA-resistant GC cells.

The target gene of miR-502-5p was searched by TargetScan. And ADAM9 was proved to be a direct target of miR-502-5p by dual-luciferase reporter assay and RIP assay. As a member of the ADAM family, ADAM9 could accelerate growth, invasion and metastasis of different types of human solid cancer. ${ }^{35}$ ADAM9 was closely associated with GC cell proliferation and invasion. Reducing ADAM9 activity might help to inhibit GC development and metastasis. ${ }^{36}$ Moreover, the tumor-suppressor role of miR-129-5 $\mathrm{p}^{37}$ or miR-126 ${ }^{17}$ in GC was attributed to targeting ADAM9. Consistent with a previous report, ${ }^{38}$ ADAM9 was upregulated in GC tissues and cells, especially in OXA-resistant tissues and cells. And hsa_circ_0000144 positively regulated ADAM9 expression by absorbing miR-502-5p. We found the overexpressed ADAM9-induced reversed impact on the repressed OXA resistance, proliferation and metastasis in OXA-resistant GC cells, which were triggered by miR-502-5p. Collectively, hsa_circ_0000144 might affect OXA resistance and progression in GC through miR-502-5p/ADAM9 pathway.

In conclusion, hsa_circ_0000144 was highly enriched in OXA-resistant tissues and cells. Hsa_circ_0000144 sponged miR-502-5p to upregulate ADAM9, thereby aggravating OXA resistance and progression of GC. We were the first to manifest the involvement of hsa_circ_0000144/miR-5025p/ADAM9 regulatory axis in GC development. Our findings highlighted a promising target for GC therapy. 


\section{Ethics Approval}

The design of this protocol follows the tenets of the Declaration of Helsinki, acquired permission from the Ethics Committee of Baoji Central Hospital and Animal studies were performed in compliance with the ARRIVE guidelines and the Basel Declaration.

\section{Acknowledgment}

These two authors contributed to this work equally as cofirst authors: Haifeng Gao, Jiajia Xu.

\section{Disclosure}

The authors declare that they have no financial or nonfinancial conflicts of interest for this work.

\section{References}

1. Siegel RL, Miller KD, Jemal A. Cancer statistics, 2019. CA Cancer J Clin. 2019;69(1):7-34. doi:10.3322/caac.21551

2. Compare D, Rocco A, Nardone G. Risk factors in gastric cancer. Eur Rev Med Pharmacol Sci. 2010;14(4):302-308.

3. Biagioni A, Skalamera I, Peri S, et al. Update on gastric cancer treatments and gene therapies. Cancer Metastasis Rev. 2019;38 (3):537-548. doi:10.1007/s10555-019-09803-7

4. Apps MG, Choi EH, Wheate NJ. The state-of-play and future of platinum drugs. Endocr Relat Cancer. 2015;22(4):R219-233. doi:10.1530/erc-15-0237

5. Chen LL, Yang L. Regulation of circRNA biogenesis. RNA Biol. 2015;12(4):381-388. doi:10.1080/15476286.2015.1020271

6. Wang KW, Dong M. Role of circular RNAs in gastric cancer: recent advances and prospects. World J Gastrointest Oncol. 2019;11 (6):459-469. doi:10.4251/wjgo.v11.i6.459

7. Wei L, Sun J, Zhang N, et al. Noncoding RNAs in gastric cancer: implications for drug resistance. Mol Cancer. 2020;19(1):62. doi:10.1186/s12943-020-01185-7

8. Shen F, Liu P, Xu Z, et al. CircRNA_001569 promotes cell proliferation through absorbing miR-145 in gastric cancer. $J$ Biochem. 2019;165(1):27-36. doi:10.1093/jb/mvy079

9. Liu YY, Zhang LY, Du WZ. Circular RNA circ-PVT1 contributes to paclitaxel resistance of gastric cancer cells through the regulation of ZEB1 expression by sponging miR-124-3p. Biosci Rep. 2019;39(12). doi: $10.1042 / \mathrm{bsr} 20193045$

10. Wu Q, Wang H, Liu L, et al. Hsa_circ_0001546 acts as a miRNA-421 sponge to inhibit the chemoresistance of gastric cancer cells via ATM/Chk2/p53-dependent pathway. Biochem Biophys Res Commun. 2020;521(2):303-309. doi:10.1016/j.bbrc.2019.10.117

11. Wei J, Wang J, Gao X, Qi F. Identification of differentially expressed circRNAs and a novel hsa_circ_0000144 that promote tumor growth in gastric cancer. Cancer Cell Int. 2019;19:268. doi:10.1186/s12935019-0975-y

12. Bartel DP. MicroRNAs: genomics, biogenesis, mechanism, and function. Cell. 2004;116(2):281-297. doi:10.1016/s0092-8674(04) 00045-5

13. Matuszcak C, Haier J, Hummel R, Lindner K. MicroRNAs: promising chemoresistance biomarkers in gastric cancer with diagnostic and therapeutic potential. World $J$ Gastroenterol. 2014;20 (38):13658-13666. doi:10.3748/wjg.v20.i38.13658

14. Wang B, Yang H, Shen L, et al. Rs56288038 (C/G) in 3'UTR of IRF-1 Regulated by MiR-502-5p promotes gastric cancer development. Cell Physiol Biochem. 2016;40(1-2):391-399. doi:10.1159/000452554
15. Duffy MJ, Mullooly M, O'Donovan N, et al. The ADAMs family of proteases: new biomarkers and therapeutic targets for cancer? Clin Proteomics. 2011;8(1):9. doi:10.1186/1559-0275-8-9

16. Zhou M, Graham R, Russell G, Croucher PI. MDC-9 (ADAM-9/ Meltrin gamma) functions as an adhesion molecule by binding the alpha(v)beta(5) integrin. Biochem Biophys Res Commun. 2001;280 (2):574-580. doi:10.1006/bbrc.2000.4155

17. Wang J, Zhou Y, Fei X, et al. ADAM9 functions as a promoter of gastric cancer growth which is negatively and post-transcriptionally regulated by miR-126. Oncol Rep. 2017;37(4):2033-2040. doi:10.3892/or.2017.5460

18. Eisenhauer EA, Therasse P, Bogaerts J, et al. New response evaluation criteria in solid tumours: revised RECIST guideline (version 1.1). Eur $J$ Cancer. 2009;45(2):228-247. doi:10.1016/j.ejca.2008.10.026

19. Song W, Qian Y, Zhang MH, et al. The long non-coding RNA DDX11-AS1 facilitates cell progression and oxaliplatin resistance via regulating miR-326/IRS1 axis in gastric cancer. Eur Rev Med Pharmacol Sci. 2020;24(6):3049-3061. doi:10.26355/ eurrev_202003_20669

20. Livak KJ, Schmittgen TD. Analysis of relative gene expression data using real-time quantitative PCR and the 2(-Delta Delta $\mathrm{C}(\mathrm{T})$ ) Method. Methods. 2001;25(4):402-408. doi:10.1006/meth.2001.1262

21. Li X, Sun XH, Xu HY, et al. Circ ORC2 enhances the regulatory effect of miR-19a on its target gene PTEN to affect osteosarcoma cell growth. Biochem Biophys Res Commun. 2019;514(4):1172-1178. doi:10.1016/j.bbrc.2019.04.188

22. Porter AG, Jänicke RU. Emerging roles of caspase-3 in apoptosis. Cell Death Differ. 1999;6(2):99-104. doi:10.1038/sj.cdd.4400476

23. Shang Q, Yang Z, Jia R, Ge S. The novel roles of circRNAs in human cancer. Mol Cancer. 2019;18(1):6. doi:10.1186/s12943-018-0934-6

24. Guo LL, Song CH, Wang P, et al. Competing endogenous RNA networks and gastric cancer. World $J$ Gastroenterol. 2015;21 (41):11680-11687. doi:10.3748/wjg.v21.i41.11680

25. Ruan Y, Li Z, Shen Y, et al. Functions of circular RNAs and their potential applications in gastric cancer. Expert Rev Gastroenterol Hepatol. 2020;14(2):85-92. doi:10.1080/17474124.2020.1715211

26. Huang W, Lu Y, Wang F, Huang X, Yu Z. Downregulation of circular RNA hsa_circ_0000144 inhibits bladder cancer progression via stimulating miR-217 and suppressing RUNX2 expression. Gene. 2018;678:337-342. doi:10.1016/j.gene.2018.08.036

27. Fang X, Bai Y, Zhang L, Ding S. Silencing circSLAMF6 represses cell glycolysis, migration, and invasion by regulating the miR-2045p/MYH9 axis in gastric cancer under hypoxia. Biosci Rep. 2020;40 (6). doi:10.1042/bsr20201275

28. Mi L, Lei L, Yin X, et al. Circ_0000144 functions as a miR-623 sponge to enhance gastric cancer progression via up-regulating GPRC5A. Biosci Rep. 2020;40(8). doi:10.1042/bsr20201313

29. Hansen TB, Jensen TI, Clausen BH, et al. Natural RNA circles function as efficient microRNA sponges. Nature. 2013;495 (7441):384-388. doi:10.1038/nature11993

30. Zhai H, Song B, Xu X, Zhu W, Ju J. Inhibition of autophagy and tumor growth in colon cancer by miR-502. Oncogene. 2013;32 (12):1570-1579. doi:10.1038/onc.2012.167

31. Sun LL, Wang J, Zhao ZJ, et al. Suppressive role of miR-502-5p in breast cancer via downregulation of TRAF2. Oncol Rep. 2014;31 (5):2085-2092. doi:10.3892/or.2014.3105

32. Ying Y, Li J, Xie H, et al. CCND1, NOP14 and DNMT3B are involved in miR-502-5p-mediated inhibition of cell migration and proliferation in bladder cancer. Cell Prolif. 2020;53(2):e12751. doi:10.1111/cpr.12751

33. Peng X, Wu M, Liu W, et al. miR-502-5p inhibits the proliferation, migration and invasion of gastric cancer cells by targeting SP1. Oncol Lett. 2020;20(3):2757-2762. doi:10.3892/ol.2020.11808

34. Yin HF, Wang Q, Huang XT, et al. Circ_0032627 accelerates proliferation of gastric cancer cells through regulating miR-502-5p expression. Eur Rev Med Pharmacol Sci. 2020;24(12):6674-6681. doi:10.26355/eurrev_202006_21654 
35. Oria VO, Lopatta P, Schilling O. The pleiotropic roles of ADAM9 in the biology of solid tumors. Cell Mol Life Sci. 2018;75 (13):2291-2301. doi:10.1007/s00018-018-2796-x

36. Kim JM, Jeung HC, Rha SY, et al. The effect of disintegrin-metalloproteinase ADAM9 in gastric cancer progression. Mol Cancer Ther. 2014;13(12):3074-3085. doi:10.1158/1535-7163. mct-13-1001
37. Liu Q, Jiang J, Fu Y, et al. MiR-129-5p functions as a tumor suppressor in gastric cancer progression through targeting ADAM9. Biomed Pharmacother. 2018;105:420-427. doi:10.1016/j. biopha.2018.05.105

38. Carl-McGrath S, Lendeckel U, Ebert M, Roessner A, Röcken C. The disintegrin-metalloproteinases ADAM9, ADAM12, and ADAM15 are upregulated in gastric cancer. Int J Oncol. 2005;26(1):17-24.

\section{Publish your work in this journal}

OncoTargets and Therapy is an international, peer-reviewed, open access journal focusing on the pathological basis of all cancers, potential targets for therapy and treatment protocols employed to improve the management of cancer patients. The journal also focuses on the impact of management programs and new therapeutic

Submit your manuscript here: https://www.dovepress.com/oncotargets-and-therapy-journal agents and protocols on patient perspectives such as quality of life, adherence and satisfaction. The manuscript management system is completely online and includes a very quick and fair peer-review system, which is all easy to use. Visit http://www.dovepress.com/ testimonials.php to read real quotes from published authors. 